\title{
Del cuidado registrado al diagnóstico enfermero
}

\author{
Manuela Domingo Pozo, Francisco Javier Gomez Robles, Maria Torres Figuelras \\ y EVA M. ${ }^{A}$ GabaLdon Bravo \\ Unidad planes de cuidados de Enfermería. Hospital General Universitario de Alicante. Alicante. España.
}

\section{Resumen}

Objetivos. Los objetivos de este studio de tipo descriptivo y analítico son: a) clasificar las expresiones utilizadas por las enfermeras en la hoja de cuidados del paciente en el Hospital General Universitario de Alicante (HGUA) en un sistema de categorías teóricas establecido por el equipo investigador en un estudio piloto; b) analizar las expresiones prediagnósticas que registran las enfermeras del HGUA, y c) inducir los diagnósticos enfermeros más frecuentes en el HGUA a través de las actividades registradas en la hoja de cuidados.

Método. Hojas de cuidados: 401. Variables: 22 categorías teóricas y expresiones prediagnósticas registradas. Análisis de contenido: expresiones agrupadas en el sistema de categorías.

Resultados. Unidades de texto: 5.589. Categorías más frecuentes: control de constantes, administración de medicación, bienestar general, pruebas diagnósticas. Se inducen 33 etiquetas diagnósticas (de 656 expresiones prediagnósticas). Más frecuentes: riesgo de infección (26,07\%), dolor agudo (19,97\%) y deterioro de la integridad tisular/cutánea $(13,11 \%)$.

El análisis de las anotaciones refleja la cultura y los valores de la profesión, considerables para el cambio de la organización de trabajo en el HGUA.

\section{Palabras clave: Análisis de contenido. Diagnósticos de enfermería. Plan de cuidados.}

Domingo Pozo M, Gómez Robles F J, Torres Figueiras M y Gabaldón Bravo E M. Del cuidado registrado al diagnóstico enfermero. Enferm Clin. 2005;15(5):00-00

\section{Abstract}

\section{From nursing care sheets to nursing diagnoses}

Objective. The aims of this descriptive analytical study were: 1) To classify the expressions used by nurses in their care plans at the Alicante General Hospital (HGUA) in a theoretical categories system designed for a pilot study by a research group. 2) To analyze the pre-diagnosis expressions used by nurses in their nursing records. 3) To identify the most frequent nursing diagnoses in the HGUA through the activities recorded in care plans.

Method. There were 401 nursing care sheets. Regarding the study variables, 22 theoretical categories and prediagnosis expressions were recorded by nurses. The contents of care plans were analyzed and expressions were grouped within the categories system.

Results. There were 5,589 entries. The most frequent categories were monitoring of vital signs, drug administration, general well-being, and diagnostic tests. Thirty-three diagnostic expressions were identified from 656 pre-diagnosis expressions analyzed. The most frequent were: risk of infection (26.07\%), acute pain (19.97\%), and deterioration of tissue/skin integrity (13.11\%).

The analysis of entries reflected the culture and values of the profession, which should be taken into consideration when implementing changes in organization of work in the HGUA.

\section{Correspondencia:}

Manuela Domingo Pozo. Avenida de los Ríos, 36 . 03699 Partida Moralet. Alicante. España.

Correo electrónico: domingo_man@gva.es 


\section{Introducción}

Los estudios de prevalencia del diagnóstico enfermero $(\mathrm{DE})^{1-7}$ comienzan a tener gran importancia en España. Si se quieren "medir los cuidados" y conocer los resultados que obtienen las enfermeras, o implantar planes de cuidados estandarizados, es imprescindible conocer los problemas más frecuentes en el medio hospitalario ${ }^{8-10}$, teniendo en cuenta limitaciones tales como el bajo nivel de cumplimentación de registros y la infrautilización del $\mathrm{DE}^{11-13}$.

A pesar de los intentos de puesta en marcha del proceso de atención de enfermería ${ }^{14-21}$, o de DE en la asistencia sanitaria, no acaba de generalizarse el uso del diagnóstico en la asistencia. Quero ${ }^{22}$ afirma la conveniencia de incluir DE independientemente de que estuvieran aprobados o no por la North American Nursing Diagnosis Association (NANDA), al objeto de facilitar su uso, comprensión y utilización.

Teixidor ${ }^{23}$ incide en que "el diagnóstico médico induce el $40 \%$ de la actividad de los cuidados de enfermería, y la prescripción médica explica sólo el 26\% de la carga de trabajo de los cuidados de enfermería". Las enfermeras consideran los diagnósticos difíciles de enunciar, no poseen formación para manejarlos, no se adaptan al contexto cultural, requieren tiempo para su formulación y planificación de actividades y no los reconocen con palabras propias ${ }^{24}$. Por otro lado, no se encuentran en condiciones de negar la utilización de la NANDA mientras no se realice un estudio previo de validación de cada categoría diagnóstica ${ }^{25}$. Debido a esto, y también al bajo nivel de cumplimentación de los registros, se realiza un estudio piloto descriptivo en el Hospital General Universitario de Alicante (HGUA) $)^{11}$ para poner de manifiesto la inexistencia de criterios unificados en terminología enfermera.

La planificación de cuidados aparece en el 2,25\% de los registros de cuidados auditados, y mayoritariamente aparecen tareas delegadas por otros profesionales, minimizando las directamente relacionadas con el cuidado. El DE aparece en el 0\% de las hojas. Además, se quiso investigar los conocimientos, actitudes y opiniones de las enfermeras sobre el proceso y el DE en cuanto a las actividades más reflejadas en los registros. Las enfermeras opinan que se registran sobre todo actividades delegadas en un $17,7 \%$. El 70,7\% de las enfermeras no utilizan los DE "porque nadie lo hace" $(38,3 \%)$, no se pueden aplicar al contexto profesional $(24,3 \%)$ o por falta de conocimientos $(22,4 \%)$. El $67,8 \%$ de las enfermeras no conoce otras clasifica- ciones diagnósticas ${ }^{26}$.

Conocer el lenguaje que utilizan las enfermeras no es tarea fácil debido al bajo nivel de cumplimentación de los registros de enfermería ${ }^{27,28}$. Las enfermeras sólo plasman por escrito una parte del trabajo, siguiendo procesos mentales desconocidos que probablemente están relacionados con la cultura y la autoestima profesional ${ }^{12}$. Incluso en las publicaciones se observa escasa valoración de las actividades propias del cuida$\mathrm{do}^{29-31}$.

Este estudio pretende llegar al DE desde anotaciones registradas con lenguaje propio de enfermería. Si se utilizan lenguajes similares al que manejan los profesionales en su trabajo diario, se minimiza el rechazo por falta de comprensión o no adecuación a la realidad de las unidades.

¿Se pueden adaptar los diagnósticos de la NANDA a la realidad española? ¿Y al lenguaje habitual de las enfermeras del HGUA?

Para responder a estos y otros interrogantes se plantean las siguientes hipótesis:

1. De las expresiones prediagnósticas registradas por las enfermeras se inducen los DE más prevalentes del HGUA.

2. Los cuidados registrados se distribuyen en torno a un sistema de categorías teóricas elaborado por el equipo investigador.

Los objetivos planteados en esta investigación son:

1. Clasificar las expresiones utilizadas por las enfermeras en la hoja de cuidados del paciente en un sistema de categorías teóricas previamente establecido por el equipo investigador.

2. Analizar las expresiones prediagnósticas que registran las enfermeras.

3. Inducir los DE más frecuentes a través de las actividades registradas en la hoja de cuidados.

\section{Método}

Tipo de estudio: cuantitativo/cualitativo retrospectivo en 2 fases: descriptiva y analítica ${ }^{32}$.

Unidades de análisis: hojas de cuidados de enfermería del HGUA.

Selección de la muestra: muestreo aleatorio simple retrospectivo (401 hojas) de las hojas de cuidados cumplimentadas por las enfermeras en 2002. En base a los datos del año 2002, se produjeron 31.290 ingresos, con un nivel de confianza al $95 \%$, un error de estimación del $2 \%$ y un error de muestreo inferior al 
3\%. El número de hojas seleccionadas es de $\mathrm{N}=401$ historias.

\section{Variables en estudio}

- Sistema de 22 categorías teóricas establecido en el estudio piloto (tabla 1$)^{11}$

- Expresiones prediagnósticas registradas por las enfermeras en la hoja de cuidados que reflejan una respuesta humana del paciente a un problema de salud/enfermedad, competencia exclusiva de la enfermería, pero sin formularlo según la clasificación de DE de la NANDA.

\section{Análisis de contenido de las hojas de cuidados}

El proceso de análisis del primer y segundo objetivos se realiza siguiendo las fases de la teoría fundamentada de Glaser y Strauss, y la codificación y el análisis se conjugan según el método de comparación constan$t^{33,34}$. Se pretende conocer qué escriben las enfermeras, cómo lo escriben y cuáles son las expresiones prediagnósticas utilizadas.

\section{Unidades de análisis}

- Unidades de registro: 22 categorías basadas en el estudio piloto previo (11). Método de tanteo (estudio piloto), siguiendo un principio de clasificación de fondo (temas) establecido por acuerdo del equipo investigador (codificación abierta). Una vez clasificadas las anotaciones en las 22 categorías establecidas por el equipo investigador, se construye una nueva categoría "expresiones prediagnósticas". Se analiza cada una de las 22 categorías para configurar la evaluación de la situación del paciente desde un punto de vista enfermero.

- Unidades de contexto: las unidades de hospitalización del HGUA (Conselleria de Sanitat de la Comunidad Valenciana), excepto urgencias, UCI, observación, reanimación, quirófanos, radiología, despertar quirúrgico y trasplantes. Duración del estudio: de noviembre de 2003 a junio de 2004.

Recogida de datos: transcripción literal de los 4 investigadores de las expresiones utilizadas por las enfermeras en una planilla de recogida de datos dividida en categorías analizadas en el estudio piloto. Se recoge todo lo escrito por las enfermeras en las primeras $24 \mathrm{~h}$ de ingreso del paciente, por ser el intervalo de tiempo de más carga de cuidados y donde se establecen los DE.

Se unificaron criterios por parte del equipo investigador y se realizó una primera prueba. El grado de concordancia en la agrupación de las expresiones dentro de las categorías entre los 4 investigadores fue muy alto. Si el observador tenía dudas a la hora de clasificar una expresión dentro de una categoría, existía la opción n. ${ }^{\circ} 22$, llamada "otras", para más tarde analizar en grupo dónde se incluiría la expresión. Todas las expresiones de esta categoría fueron analizadas por los investigadores y, por consenso, se englobaron en otras categorías.

Análisis de datos: análisis de contenido apoyado en el software Nudist-5. Se realiza un análisis de contenido semántico.

\section{Resultados}

Anotaciones registradas: 5.589 unidades de texto.

La distribución de las expresiones en el sistema de categorías propuesto se muestra en la tabla 2. Las categorías más frecuentes son control de constantes, administración y preparación de medicación, bienestar general, pruebas diagnósticas, cuidados relacionados con la alimentación, expresiones de motivo de ingreso o diagnósticos médicos, dolor y analgesia, consultas a otros profesionales y control de catéteres.

Las referencias a la familia en los registros suponen el 0,24\% y aparecen en la categoría aspectos psicológicos y consultas a otros profesionales. El término utilizado es "familia" o "familiar". El término "paciente" aparece 67 veces $(1,3 \%)$. No se ha encontrado ningún DE de la NANDA (0\%) en la hoja de cuidados.

Tras analizar las 5.589 unidades de texto, 656 se han considerado germen de DE (12\%). Las etiquetas diagnósticas extraídas de las expresiones prediagnósticas son 33 (tabla 3), y las más frecuentes son: riesgo de infección $(26,07 \%)$, dolor agudo $(19,97 \%)$ y deterioro de la integridad cutánea/tisular $(13,11 \%)$. Los déficit de autocuidado son bajos (3,51, 3,05 y 0,76\%).

Se han hallado 2 expresiones prediagnósticas ("regular estado general" y "estado delicado") imprecisas para determinar una equivalencia con etiquetas de la NANDA.

A continuación se presentan las expresiones más utilizadas por categoría:

- Control de constantes. Las más medidas son la temperatura y la presión arterial. Las frecuencias cardíaca $(1,7 \%)$ y respiratoria $(0,32 \%)$ apenas se reflejan. El término "afebril" (20\%) y "apirético" (2,2\%) son los más utilizados para referirse a la temperatura. "Normotenso" (13\%) y "PA = 170/90" $(19 \%)$ son las 
más utilizadas para la presión arterial. Poco uso de las unidades de medida ( $\mathrm{mmHg}$ sólo se utiliza en el 1,4\%). La abreviatura " $\mathrm{T}$ " aparece 103 veces.

- Administración y preparación de medicación. Refieren medicamentos administrados, y sólo hay 3 expresiones para reacciones a la medicación $(0,64 \%)$. Los verbos más utilizados son "poner", "dar" y "administrar". El término "tto" aparece 97 veces (14\%).

- Bienestar general. El término "bien" (44\%) para explicar el estado del paciente. La expresión "pasa bien la mañana/tarde/noche" aparece de forma rutinaria (24\%) y "sin incidencias" (6,9\%). "BEG", abreviatura de buen estado general, supone el $11 \%$ de las unidades de texto dentro de esta categoría.

- Pruebas diagnósticas. Referidas a pruebas solicitadas, cursadas o realizadas por la enfermera. "AS", abreviatura de analítica de sangre (11\%).

- Cuidados referidos a la alimentación. Relacionadas con la tolerancia oral (18\%), la dieta o si está en ayunas. Utilización del signo + para indicar tolerancia positiva del paciente $(4,7 \%)$.

- Expresiones, motivo de ingreso, traslado, diagnósticos médicos. Recoge los movimientos del paciente por el hospital, como "ingreso" (29\%) y "traslados" (7\%). También engloba diagnósticos médicos (hipertenso, DM, ACV...).

- Dolor y analgesia. Dos expresiones que reflejan medidas o cuidados no farmacológicos para el dolor: "poner almohada por dolor en la rodilla y cede", "poner hielo por dolor en la episiotomía". Veintitrés expresiones referidas a efectividad de la analgesia administrada. La mayoría de expresiones se refieren a administración de analgésicos del tipo "pongo $\mathrm{X}$ fármaco", "doy X fármaco". La palabra "dolor" supone el $50 \%$ de las unidades de texto.

- Consultas a otros profesionales. Expresiones "visto/a X médico" (37\%), "Aviso a..."(8\%).

- Control de catéteres. La mayoría referidas a canalizar, poner o cambiar vía, más que a cuidados requeridos por los catéteres. Términos como "vía" (37\%) y abreviaturas como "BH" (bránula heparinizada, $16 \%)$.

- Cuidados referidos a la eliminación urinaria. Versan sobre la sonda vesical (17\%), la medición o control de diuresis $(14 \%)$ y la micción tras algún tipo de anestesia (17\%).

- Cuidados quirúrgicos. Escasas alusiones a cuidados previos a la intervención. Términos más relacionados con la hora que sube o baja a quirófano o la programación; "quirófano" aparece 55 veces (21\%).
- Cuidados maternoinfantiles. Existen 58 referencias a la lactancia, 16 expresiones referidas al sangrado $(7,5 \%)$ y 13 "loquios normales" (6,1\%).

- Cuidados referidos a la respiración. Cuarenta y seis expresiones referidas a oxigenoterapia, 39 a saturación y 12 a la tos. La aspiración de secreciones, clap ping o lavados nasales sólo aparecen 10 veces. El término $\mathrm{O}_{2}$ tiene una frecuencia de $17 \%$.

- Cuidados referidos al descanso. La mayoría de expresiones son del tipo "duerme", "dormido", "durmiendo" (45), y sólo 6 expresiones hacen referencia a problemas con el descanso-sueño ("potroso", "se despierta con facilidad", "duerme poco", "insomnio", "no duerme nada", "molesto").

- Cuidados referidos a la seguridad. "Consciente y orientada" (17\%) y otros términos como "agitado" (4\%), "desorientado" (1,3\%), "inquieto" y "tranquilo" (1,3\%). Las medidas de seguridad registradas para evitar lesiones en el paciente: "barandilla", "atado", "sujeción", "vigilancia por familiar". Sólo en 2 ocasiones se utiliza una escala para medir el estado de consciencia.

- Cura de heridas. Ninguna expresión referida a la evolución o al estado de la herida.

- Cuidados referidos a la eliminación fecal. La mayoría refieren control de deposiciones, número y características. Dieciocho expresiones referidas a la administración de enemas (10\%), pero sólo 2 reflejan si es efectivo.

- Cuidados referidos a la movilidad. No existen expresiones que utilicen escalas para medir el grado de movilidad.

- Aspectos psicológicos y sociales. Utilizan expresiones del tipo "ansioso", "energúmeno", "animado", "decaído", "nervioso".

- Estado de la piel y las mucosas. Ninguna expresión para hacer referencia al riesgo de úlceras por presión.

- Otras. En esta categoría hay 26 expresiones que no se pudieron clasificar en otros apartados.

- Alergias. La expresión más frecuente es "no alergias", "no RAM".

- Aseo e higiene. De las 8 expresiones registradas para referirse a la higiene del paciente, en 4 se refieren a la realización de la misma.

Expresiones prediagnósticas: el tipo de expresiones prediagnósticas clasificadas o inducidas para cada etiqueta de la NANDA se especifican en la tabla 3.

Las hojas de cuidados reflejan terminología telegráfica y muchas abreviaturas, no todas correctas: un 
lenguaje irreconocible por profesionales ajenos a la enfermería. Se utilizan expresiones muy breves cargadas de gran significado, como "mañana AS", "barandilla", "hoy Qx".

Dada la gran cantidad de datos, se procede al análisis de las etiquetas diagnósticas más frecuentes en los registros.

- Riesgo de infección (26,7\%). Relacionados con presencia de catéter en el paciente o la actividad de insertar un catéter: "BH", "cogida vía", "dejo BH", "lleva vía”, "se canaliza vía", "vía periférica”. Las expresiones referidas a cuidados de mantenimiento de los catéteres: "heparinizo vía", "periférica curada y permeable para medicar", "se cura vía". Aunque la enfermera no manifieste explícitamente el riesgo de infección en los registros, considera relevante la presencia de catéteres.

- Dolor agudo (19,97\%). Expresiones que contienen "dolor", referidas o bien a la administración de analgesia por dolor ("puesta analgesia x dolor", "precisa calmantes", " 5 h dolor puesto nolotil iv") o son valoraciones del dolor del paciente ("le duele bastante", "molesto durante la tarde", "dolor controlado", "avisa por dolor"). No aparecen escalas de valoración del dolor.

- Deterioro de la integridad tisular/cutánea $(13,11 \%)$. Escasas referencias a la localización y evolución de la herida. Informan de la presencia de algún tipo de herida o erosión: "colostomía", "episiotomía", "erosiones diversas por todo el cuerpo", "escara en cadera", "úlceras en talones", "MI edematizados". Otras referidas a la acción de curar, pero no con qué, ni cómo: "curado", "curada por manchar bastante", "cura local diaria", "está curada escara". Las expresiones "se le cambia vía por flebitis" y "cogemos vía de nuevo pues se le ha extravasado" indican deterioro de la integridad tisular y son términos más cercanos que la etiqueta diagnóstica.

- Déficit de autocuidados: baño/higiene (3,51\%). Alusión a presencia de pañales o higiene: "cambiados pañales", "pañal" y "realizada higiene". Uso WC $(3,05 \%)$. Alimentación (0,76\%). "Cubiertas necesidades básicas" y "dependiente para realizar funciones básicas” son expresiones que indican que el paciente tiene un déficit de autocuidados.

- Hipertermia (3,35\%). "Fiebre", "llega con fiebre de $38^{\circ}$."

- Confusión aguda (3,2\%). "Agitación", "desorientado", "alteración del nivel de conciencia". Llama la atención "barandillas" con carga de significado.
- Náuseas (3,2\%). "Está con náuseas y vómitos" o "vomita".

- Lactancia materna eficaz (3,2\%). "Lactancia materna bien", "toma muy bien el bebé".

- Riesgo de caídas (2,13\%). "Se marea y casi se cae al suelo"; alusiones a medidas estrictas de seguridad: "sujeciones físicas + barandillas"; este tipo de medidas puede deberse tanto a un riesgo de caídas como a confusión aguda.

- Estreñimiento (1,98\%). "No deposiciones", "pautan laxante".

- Deterioro de la movilidad física (1,98\%). "Precisa cambios posturales", que indica déficit de autocuidados.

- Ansiedad (1,83\%). Utilización de adjetivos como "ansioso", "inquieto", "intranquilo", "nervioso".

- Patrón respiratorio ineficaz (1,68\%). "Fatiga", "disnea".

- Retención urinaria (1,37\%). "Se coloca sonda vesical por globo de retención".

- Limpieza ineficaz de las vías aéreas (1,22\%). "Aspiro secreciones", "hacemos claping".

- Incontinencia fecal/urinaria (0,46\%). "Con pañales por incontinencia urinaria", "incontinencia fecal (lleva pañal)".

\section{Discusión}

Se cumplen los objetivos y se confirman las hipótesis del estudio. En la tabla 3 se observan los DE más frecuentes en el HGUA. El riesgo de infección, el dolor agudo y el deterioro de la integridad tisular/cutánea son los más detectados y registrados, y coinciden con los indicadores de calidad de los cuidados que actualmente se están midiendo: tasas de flebitis, infecciones urinarias por sondaje vesical, úlceras por presión y dolor. Los diagnósticos derivados de problemas de autonomía o de carácter psicológico como ansiedad, temor o afrontamiento ineficaz apenas son motivo de registro.

De las 5.589 unidades de texto analizadas, ninguna se corresponde con un DE de la NANDA (0\%), dato en contradicción con la respuesta de las enfermeras, que afirman utilizarlos en un $29 \%{ }^{26}$. Puede deberse al desconocimiento de los diagnósticos NANDA, o a que las enfermeras no reconocen su lenguaje en los diagnósticos de la NANDA.

Se han encontrado 2 expresiones ("regular estado general" y "estado delicado") que, por su imprecisión, 
no han podido clasificarse con ninguna etiqueta.

Respecto a la segunda hipótesis, el sistema de categorías presentado es válido para agrupar las anotaciones de las enfermeras; para una validación externa habría que continuar el estudio con grupos nominales, uno por categoría, y analizar si las enfermeras reconocen las expresiones dentro de cada categoría.

Cabe destacar que algunas actividades realizadas, que son competencia exclusiva enfermera, no se registran. Así, el término "hoy Qx" llevaría implícito una serie de cuidados de enfermería (preparación quirúrgica) que no se registran de manera explícita.

Las referencias a la familia en los registros son anecdóticas. El término utilizado es "familia" o "familiar", sin aclarar grado de parentesco o relación con el paciente.

No se han encontrado estudios similares que permitan comparar los resultados. La mayoría de los estudios de prevalencia de problemas de enfermería se realizan tras un proceso de estandarización de cuidados, normalmente informatizado, donde la enfermera marca la etiqueta diagnóstica. Uno de los estudios ${ }^{1}$, tras la estandarización informatizada de los planes de cuidados, en los informes de alta, coincide en que uno de los diagnósticos de enfermería más frecuente es "riesgo de infección" (66,5\%), y otro ${ }^{7}$ coincide en sus conclusiones en que los problemas más registrados son de la esfera biomédica, mientras que los problemas del área psicosocial apenas aparecen.

En cuanto a las limitaciones del estudio, cabe resaltar que los datos obtenidos en torno a los DE de la taxonomía NANDA, extraídos de las expresiones denominadas "prediagnósticas" por el equipo investigador, no pueden generalizarse a otros contextos sin estudios que adecuen la situación de los registros. Los hallazgos obtenidos con esta investigación permiten al HGUA reflexionar sobre los problemas/diagnósticos de enfermería con más carga de registro y, por tanto, de incidencia en la carga de cuidados.

\section{Agradecimientos}

Gracias a D. Miguel Ángel Fernández Molina por su inestimable apoyo y facilitador del acceso al campo de estudio. Gracias a M. ${ }^{a}$ José Muñoz por su ayuda con el idioma. Gracias también a Dr. D. Eleuterio Gandía por sus tutorías de parte de este trabajo.

\section{Bibliografía}

1. Rodríguez B, Ferrer MA. Prevalencia de diagnósticos enfermeros de la NANDA y necesidades alteradas de Henderson en una unidad de lactantes. Enferm Clin. 2002;12:253-9.

2. Botas B, Rivas C, Velado ML, Linares Y, Muñoz R. Prevalencia de diagnósticos de enfermería en una unidad neonatal. Enferm Clin. 2001;11:9-17.

3. Clavería M. Diagnósticos de enfermería utilizados por un equipo de atención domiciliaria socio-sanitaria. Enferm Cient.1997:182-3:49. 54.

4. Guirao J. Diagnóstico de enfermería en una consulta de enfermería de atención primaria. Estudio descriptivo. Enferm Integral. 1993;26:2734.

5. De Pedro J, Gallo J, Zaforteza C, Bover J, Galmes A. Prevalencia de los diagnósticos enfermeros en el paciente geriátrico después del alta y su evolución. Rev Multidiscip Gerontol. 2001;11:20-2.

6. Fernández C, Román E, Martínez R. Método de trabajo en una institución geriátrica basado en los diagnósticos enfermeros. Gerokomos. 2001;12:107-13.

7. Nieto E, Ramos O. Evolución del lenguaje enfermero: evaluación de los diagnósticos en un informe de alta. Metas Enferm. 2000;22:45-52.

8. Serrano R, Narvaiza MJ, Saracíbar MI, Carrascal E, Tina P, Sanz M, et al. Correspondencia entre los problemas de salud y los cuidados de enfermería al ingreso del paciente. Enferm Clin. 2000;10:47-56.

9. Duarte G, Montesinos N. El consenso como estrategia de implementación del diagnóstico enfermero, valoraciones estandarizadas y selección de etiquetas diagnósticas. Enferm Clin.1999;9:142-50.

10. Rodríguez MM, Torrés A, Azañón R, Rodríguez M, Palma J. Experiencia en la implantación del proceso enfermero en emergencias. Tempus Vitalis. 2003;3:4-10.

11. Gómez J, Domingo M, Pozo EM, Gabaldón C, Terrés M, Torres M, et al. Terminología utilizada por las enfermeras del Hospital General de Alicante en la hoja de cuidados de enfermería. Index Enferm. 2003; 40-1:16-9.

12. Gabaldón E, Gómez J, Fernández M, Domingo M, Torres M, Terrés $\mathrm{C}$, et al. Análisis de las causas del bajo nivel de cumplimentación del paciente al ingreso: una aproximación desde la perspectiva cualitativa. Cult Cuid. 2002;4:53-60.

13. Serrano Sastre R, Saracíbar Rázquin MI, Díaz Narvalaz MT, Tina Majuelo P, Narvaiza Solís MJ. ¿Dónde comienza la calidad científica de los documentos de enfermería? El reto de la documentación. Rev Rol Enferm. 1994;191-2:33-40.

14. Llamas Urrutia C. ¿Cómo se utiliza el proceso de cuidados de enfermería? Rev Rol Enf 2003;26:354-62.

15. Sánchez A, Sans C. Protocolizar las actividades de enfermería. Rev Rol Enf. 2001;24:67-74.

16. Serrano R, et al .Estandarizar los cuidados, cuando lo hecho es válido. Rev Rol Enf.1997;227-8:23-31.

17. Carbelo B, et al. Estrategias de implementación de un plan de cuidados estandarizado en una unidad de hospitalización médica. Enf Cientif.1997;188-9:78-82.

18. González $\mathrm{P}$, et al. Validación de los diagnósticos de enfermería de la NANDA: una experiencia en cuatro áreas asistenciales. Enferm Clin. 1995;4:124-7.

19. Gordon M. Pasado, presente y futuro de los diagnósticos de enfermería. Cult Cuid. 2000;7-8:128-38.

20. Feria D, Macías AC, Romero M, Mena FJ, Valderrama A. Valoración del método de trabajo que utilizan los profesionales de enfermería en el ámbito hospitalario del Servicio Andaluz de Salud. Metas Enferm. 2000;111:41-8.

21. Maña M, Sáez A. Resultados obtenidos en consulta de enfermería tras la protocolización de planes de cuidados. Experiencia en un EAP de Torrejón de Ardoz. Enferm Cient. 1997;184-5:34-9.

22. Quero A. El diagnóstico de enfermería. Una aproximación a la realidad española. Index Enferm.1995;12-3:24-7.

23. Teixidor M. El producto enfermero y la reforma de los procesos asistenciales (III). Rev Rol de Enf. 2002;25:408-10.

24. Izquierdo JM, Pérez MB, Ramírez FJ, Serrano I, Torres MD, Conde G. Implantación del proceso enfermero. Rev Rol Enf. 2002;25:488-93.

25. Luis MT, González P, Germán C, Coscolla E, Benavent A, Palomino 
P, et al. El diagnóstico de enfermería en España. ¿Una realidad apreciable? Index Enferm. 1995;12-3:14-23.

26. Domingo M, Gómez FJ, Ballesta F, Torres M, et al. Opinión de las enfermeras del Hospital General Universitario de Alicante sobre la implantación de planes de cuidados. Comunicación presentada en las IX Jornadas de Enfermería del HGUA. Alicante; noviembre 2003.

27. Fernández MA, Gómez J, Gabaldón EM, Domingo M, Torres M, Terrés C. Evaluación del impacto de las intervenciones realizadas para mejorar el registro de valoración de enfermería al ingreso. Enferm Clin. 2003;13:137-44

28. Martínez C, Cañadas A, Rodríguez E, Tornel S. Dificultades en la valoración enfermera. Enferm Clin. 2003;13:195-201.

29. Nieto E, Domingo R. Representación social de la guía metodológica de enfermería que tienen las enfermeras del área XI de atención primaria (Madrid, INSALUD). Disponible en: http://www.arrakis.es/ jsacris/trabajo.html.

30. Mena FJ, Macías AC, Romero M, Valderrama A, Feria D. :Influyen los diagnósticos de enfermería en la valoración del método de trabajo enfermero? Rev Rol Enf. 2001;24:137-9.

31. Ortega C, Sánchez N. Lenguaje de enfermería en las publicaciones científicas. Enferm Cient.1999;xxx:206-7.

32. Pino R, Ugalde M. Líneas de investigación en diagnósticos enfermeros. Enferm Clin.1999;9:115-20.

33. Glaser B, Strauss A. The discovery of Grounded Theory. Chicago: Aldine; 1967.

34. García M, Ibáñez J, Alvira F. El análisis de la realidad social. Métodos y técnicas de investigación. 3. ${ }^{a}$ ed. Madrid: Alianza; 2000. 
Domingo Pozo M, et al. Del cuidado registrado al diagnóstico enfermero

\section{TABLA 1. Variables del sistema de categorías}

Pruebas diagnósticas

Cuidados quirúrgicos

Cuidados de catéteres

Bienestar general

Administración y preparación de medicación

Control de constantes vitales

Consultas a otros profesionales

Cuidados referidos a aseo e higiene

Cuidados referidos a la alimentación

Cuidados referidos al dolor

Cuidados referidos a la seguridad

Cuidados referidos a la eliminación urinaria

Cuidados referidos a la eliminación fecal

Cuidados referidos al descanso

Cuidados referidos a la respiración

Cuidados referidos a la movilidad

Cuidados maternoinfantiles

Cura de heridas

Aspectos psicosociales

Alergias

Estado de piel y mucosas

Otras 


\section{TABLA 2. Distribución de las categorias por frecuencias}

\begin{tabular}{|c|c|c|}
\hline Sistema de categorias & $\begin{array}{c}\text { Frecuencia } \\
\text { absoluta }\end{array}$ & $\%$ \\
\hline Control de constantes & 630 & 11,27 \\
\hline Administración y preparación de medicación & 471 & 8,42 \\
\hline Bienestar general & 376 & 6,72 \\
\hline Pruebas diagnósticas & 365 & 6,53 \\
\hline Cuidados relacionados con la alimentación & 328 & 5,86 \\
\hline Expresiones "motivo ingreso", "traslado", "diagnóstico médico" & 287 & 5,14 \\
\hline Dolor y analgesia & 189 & 3,38 \\
\hline Consultas a otros profesionales & 188 & 3,36 \\
\hline Control de catéteres & 187 & 3,35 \\
\hline Cuidados referidos a la eliminación urinaria & 167 & 3 \\
\hline Cuidados quirúrgicos & 161 & 2,88 \\
\hline Cuidados maternoinfantiles & 137 & 2,45 \\
\hline Cuidados referidos a la respiración & 131 & 2,35 \\
\hline Cuidados referidos al descanso & 107 & 1,91 \\
\hline Cuidados referidos a la seguridad & 81 & 1,45 \\
\hline Cura de heridas & 80 & 1,43 \\
\hline Cuidados referidos a la eliminación fecal & 60 & 1,07 \\
\hline Cuidados referidos a la movilidad & 60 & 1,07 \\
\hline Aspectos psicológicos y sociales & 32 & 0,57 \\
\hline Estado piel y mucosas & 28 & 0,50 \\
\hline Otras & 26 & 0,47 \\
\hline Alergias & 24 & 0,43 \\
\hline Aseo e higiene & 8 & 0,14 \\
\hline
\end{tabular}


Domingo Pozo M, et al. Del cuidado registrado al diagnóstico enfermero

\begin{tabular}{|c|c|c|}
\hline Etiquetas diagnósticas detectadas & Frecuencia absoluta & $\%$ \\
\hline Riesgo de infección & 171 & 26,07 \\
\hline Dolor agudo & 131 & 19,97 \\
\hline Deterioro de la integridad cutánea/tisular & 86 & 13,11 \\
\hline Déficit de autocuidados: baño e higiene & 23 & 3,51 \\
\hline Hipertermia & 22 & 3,35 \\
\hline Confusión aguda & 21 & 3,2 \\
\hline Náuseas & 21 & 3,2 \\
\hline Lactancia materna eficaz & 21 & 3,2 \\
\hline Déficit de autocuidados: uso del WC & 20 & 3,05 \\
\hline Riesgo de caídas & 14 & 2,13 \\
\hline Estreñimiento & 13 & 1,98 \\
\hline Deterioro de la movilidad física & 13 & 1,98 \\
\hline Ansiedad & 12 & 1,83 \\
\hline Patrón respiratorio ineficaz & 11 & 1,68 \\
\hline Retención urinaria & 9 & 1,37 \\
\hline Limpieza ineficaz de las vías aéreas & 8 & 1,22 \\
\hline Riesgo de deterioro de la integridad cutánea/tisular & 7 & 1,07 \\
\hline Déficit de autocuidados: alimentación & 5 & 0,76 \\
\hline Diarrea & 5 & 0,76 \\
\hline Riesgo de violencia dirigida a otros/autodirigida & 5 & 0,76 \\
\hline Desequilibrio nutricional por defecto & 5 & 0,76 \\
\hline Lactancia materna ineficaz & 4 & 0,61 \\
\hline Incontinencia urinaria & 3 & 0,46 \\
\hline Incontinencia fecal & 3 & 0,46 \\
\hline Deterioro de la eliminación urinaria & 3 & 0,46 \\
\hline Trastorno de la percepción sensorial: táctil & 3 & 0,46 \\
\hline Deterioro del patrón del sueño & 2 & 0,3 \\
\hline Impotencia & 2 & 0,3 \\
\hline Baja autoestima situacional & 2 & 0,3 \\
\hline Temor & 2 & 0,3 \\
\hline Riesgo de estreñimiento & 1 & 0,15 \\
\hline Riesgo de soledad & 1 & 0,15 \\
\hline Riesgo de lesión (hemorragia) & 1 & 0,15 \\
\hline
\end{tabular}

